\title{
Dynamic Markov Random Field Model for Visual Tracking
}

\author{
Daehwan Kim ${ }^{1}$, Ki-Hong Kim ${ }^{1}$, Gil-Haeng Lee ${ }^{1}$, and Daijin Kim² \\ ${ }^{1}$ Creative Content Research Laboratory, ETRI, Daejeon, Republic of Korea \\ \{daehwank, kimgh, ghlee\}@etri.re.kr \\ 2 Department of Computer Science and Engineering, POSTECH, Pohang, Republic of Korea \\ dkim@postech.ac.kr
}

\begin{abstract}
We propose a new dynamic Markov random field (DMRF) model to track a heavily occluded object. The DMRF model is a bidirectional graph which consists of three random variables: hidden, observation, and validity. It temporally prunes invalid nodes and links edges among valid nodes by verifying validities of all nodes. In order to apply the proposed DMRF model to the object tracking framework, we use an image block lattice model exactly correspond to nodes and edges in the DMRF model and utilize the mean-shift belief propagation (MSBP). The proposed object tracking method using the DMRF surprisingly tracks a heavily occluded object even if the occluded region is more than $70 \sim 80 \%$. Experimental results show that the proposed tracking method gives good tracking performance even on various tracking image sequences(ex. human and face) with heavy occlusion.
\end{abstract}

Keywords: Markov random field, Dynamic Markov random field, Visual tracking.

\section{Introduction}

Visual tracking is an important research topic in the computer vision field since it can be applied to many applications such as surveillance, security system, augmented reality and so on. Although many tracking methods [1] [2] [3] have been proposed, it has still several problems such as pose variations, appearance changes by illumination changes, and temporal or partial occlusion. Especially, tracking heavily occluded objects is one of the most difficult problems due to the lack of their visible area and very similar appearance within the occlusion.

Depth-based tracking methods [4] and [5] track occluded objects using depth information using multiple calibrated images. While these tracking methods tracked occluded objects well, they required additional time to calibrate multiple images and to generate depth images. Appearance-based tracking methods [6] and [3] track occluded objects by searching the object appearance in the input image. Some adaptive appearance-based methods attempt to solve the occlusion problem by statistical analysis [7] [8]. While these tracking methods tracked occluded objects well and were quite insensitive to appearance changes over time, they missed occluded objects when the object appearance was very simple or repetitive.

A. Fusiello et al. (Eds.): ECCV 2012 Ws/Demos, Part III, LNCS 7585, pp. 203-212, 2012.

(C) Springer-Verlag Berlin Heidelberg 2012 
Most of those visual tracking methods under heavy occlusion only give a rough boundary box or position due to using an estimation way based on velocity or acceleration. So these do not provide exact tracking results. It is originated from non-use of local observation information in small image blocks. However, it is still very difficult to practically use them due to lack of discriminative feature of local image blocks with very similar appearance. Fortunately, a well-encoded prior knowledge [9] [10] [11] can be a good counterproposal to make discrimination of the local feature higher. It is based on constrained pairwiseness of the structured spatial arrangement of nodes in the Markov random field (MRF) model. The MRF parameters are estimated using belief propagation (BP) [12] [13] [14] [9], which is a powerful method for computing marginal distribution of hidden random variables. These methods overcome a difficulty of the multi-modal posterior distribution caused from highly similar appearance. The combination of the MRF and BP gives a good local evidence.

However, it only works when propagations among nodes of the structured layout occur correctly. Heavy occlusion causes a global drift or distortion of the whole object layout by propagating wrongly from nodes corresponding to occluded image blocks. To avoid the bad propagation with keeping the structural layout, it rather prunes the invalid nodes from the graphical structure under such a situation. Figure 1 shows the tracking examples using the conventional MRF-based tracking method [9] with two different image block lattices. The tracking result of using the pruned lattice model is better than the result of using the full lattice model. This informs that there exits effects of nodes corresponded to the occluded image blocks and the propagations from such nodes are unnecessary.

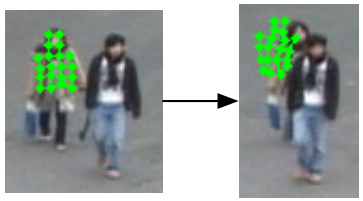

(a)

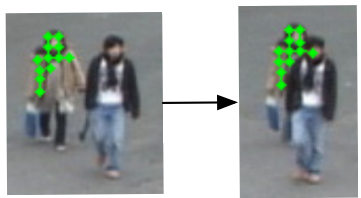

(b)

Fig. 1. Tracking examples using two different image block lattices. (a) By using human-like lattice model. (b) By using its pruned lattice model.

In this paper, we propose a dynamic Markov random field (DMRF) model that does not propagate bad effects from the invalid nodes by dynamically reconfiguring the graphical structure. The proposed model is represented by adding a validity variable to the conventional MRF model and altering an undirected edge into an bidirectional edge, respectively. The added validity variable determines whether each node is valid or not, and the altered bidirectional edge gives a permission to propagate the messages derived from its neighboring nodes. These make a structured layout of our proposed model be easily re-configured. To efficiently infer the hidden variables of the DMRF model, we use mean-shift belief propagation (MSBP) proposed by [9] to efficiently infer hidden random variables in our DMRF model. Finally, we develop an object tracking algorithm that can track a heavily occluded object even. To evaluate the tracking accuracy of the 
proposed method, we apply our approach to various object tracking image sequences including occluded human and face.

\section{Dynamic Markov Random Field}

A DMRF is a bidirectional graph $G=(V, E)$, where $V$ and $E$ represent the set of nodes and edges in the graph, where each node represents a random variable and each edge represents a statistical dependency between random variables by the connected edges. The DMRF model has three random variables: the hidden random variables $\mathbf{x}_{i}, i=$ $1,2, \cdots, N$, the observation random variables $z_{i}, i=1,2, \cdots, N$, and the validity variables $v_{i}, i=1,2, \cdots, N$, where $N$ is the number of nodes (Fig. 2).

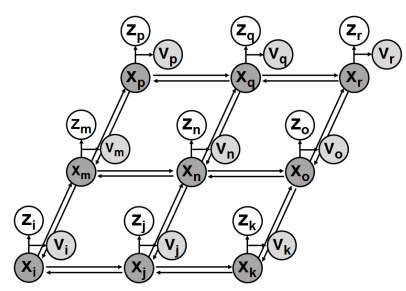

Fig. 2. A graphical structure of DMRF model

We can represent the DMRF in terms of the joint probability as

$$
p\left(\mathbf{x}_{1}, . ., \mathbf{x}_{N}, z_{1}, . ., z_{N}, v_{1}, . ., v_{N}\right)=\prod_{(i, j) \in E} \psi\left(x_{i}, x_{j}\right) \prod_{i \in N}^{N} \phi\left(x_{i}, z_{i}, v_{i}\right),
$$

where $N, \mathbf{x}_{i}, z_{i}$ and $v_{i}$ are the number of nodes, the $i$ th hidden random variable, the $i$ th observation random variable, and the $i$ th validity variable, respectively.

The pairwise compatibility function $\psi\left(\mathbf{x}_{i}, \mathbf{x}_{j}\right)$ is defined as a measurement of the spring energy between two connected nodes $\mathbf{x}_{i}$ and $\mathbf{x}_{j}$. If the value of the pairwise compatibility function becomes large, the two nodes are highly dependent on each other. In this work, we take the pairwise compatibility function as

$$
\psi\left(\mathbf{x}_{i}, \mathbf{x}_{j}\right)=\exp \left(-\alpha\left|d\left(\mathbf{x}_{i}, \mathbf{x}_{j}\right)-d\left(\mathbf{x}\left(T_{i}\right), \mathbf{x}\left(T_{j}\right)\right)\right|\right),
$$

where $\alpha$ adjusts the elasticity of the edge between two nodes, $\mathbf{x}_{i}$ and $\mathbf{x}_{j}$ denote the positions of input image blocks for the $i$ th and $j$ th nodes, respectively, $\mathbf{x}\left(T_{i}\right)$ and $\mathbf{x}\left(T_{j}\right)$ denote the positions of template image blocks for the $i$ th and $j$ th nodes, respectively, and $d(\bullet, \bullet)$ is a Euclidean distance between the positions for two nodes.

The joint compatibility function $\phi\left(\mathbf{x}_{i}, z_{i}, v_{i}\right)$ associates the probabilistic relation among a hidden random variable $\mathbf{x}_{i}$, observation random variable $z_{i}$, and its validity random variable $v_{i}$. In this work, we take the joint compatibility function as

$$
\phi\left(\mathbf{x}_{i}, z_{i}, v_{i}\right)=1-\phi_{v}\left(\mathbf{x}_{i}, v_{i}\right)\left(1-\phi_{o}\left(\mathbf{x}_{i}, z_{i}\right)\right),
$$


where, $\phi_{v}\left(\mathbf{x}_{i}, v_{i}\right)$ and $\phi_{o}\left(\mathbf{x}_{i}, z_{i}\right)$ are validity and observation compatibility functions, respectively.

The observation compatibility function $\phi_{o}\left(\mathbf{x}_{i}, z_{i}\right)$ is defined as

$$
\phi_{o}\left(\mathbf{x}_{i}, z_{i}\right)=\exp \left(-\beta \sum_{j \in W(i)}\left(I\left(\mathbf{x}_{i, j}\right)-T\left(\mathbf{x}_{i, j}\right)\right)^{2}\right),
$$

where $I$ and $T$ denote the image and template image block, respectively, $W(i)$ is the sample with the sample window for the $i$ th node, $\mathbf{x}_{i, j}$ is the $j$ th sample within the sample block for the $i$ th node, and $\beta$ is a normalizing parameter.

The validity compatibility function $\phi_{v}\left(\mathbf{x}_{i}, v_{i}\right)$ is defined as

$$
\phi_{v}\left(\mathbf{x}_{i}, v_{i}\right)=\left\{\begin{array}{l}
1, \text { if } \exp \left(-\gamma d_{v}\left(I\left(\mathbf{x}_{i, j}\right), T_{i, j},\right)\right)>t h_{v} \\
0, \text { otherwise }
\end{array}\right.
$$

where $d_{v}$ is the similarity function between a target image template block $T_{i}$ and $I\left(\mathbf{x}_{i}\right)$ (centered at location $\mathbf{x}_{i}$ of the input image). The parameter $\gamma$ adjusts the influence of the validity variable $v_{i}$ for validating an image block. $t h_{v}$ denotes the threshold value for the validity variable. Here, we used the Bhattacharyya distance as a similarity function. The reason for formulating the validity variable as Eq. (5) is as follows. When a node is valid, the value of the validity variable is equal to 1 because the DMRF should be functioned as the MRF. When a node is invalid, the value of the validity variable is equal to the reciprocal of the joint compatibility function because the right-hand product terms in Eq. (1) should be equal to 1 to make the joint compatibility function ineffective.

There are two well-known inference rules to infer the hidden random variables: SumProduct and Max-Product rules. We take the Sum-Product rule that are based on discrete belief propagation (DBP) because it is easily computed using the element-wise product of arrays [9]. We modify the existing inference rule to compute the hidden random variables in the DMRF as follows.

\subsection{Sum-Product Rule}

The sum-product rule computes the marginal posterior probability (belief) as

$$
b\left(\mathbf{x}_{i}\right)=k \phi\left(\mathbf{x}_{i}, z_{i}, v_{i}\right) \prod_{j \in N_{v}(i)} m_{j \rightarrow i}\left(\mathbf{x}_{i}\right),
$$

where $k$ and $N_{v}(i)$ are a normalization constant and the neighboring valid nodes of the $i$ th node, respectively, and $\phi\left(\mathbf{x}_{i}, z_{i}, v_{i}\right)$ is the joint compatibility function derived from only valid nodes between the image block and the template image block.

The message $m_{j \rightarrow i}\left(\mathbf{x}_{i}\right)$ passing from the $j$ th node to the $i$ th node is computed recursively as

$$
m_{j \rightarrow i}\left(\mathbf{x}_{i}\right)=\sum_{\mathbf{x}_{j}} \phi\left(\mathbf{x}_{j}, z_{j}, v_{j}\right) \psi\left(\mathbf{x}_{j}, \mathbf{x}_{i}\right) \prod_{k \in N_{v}(j) \backslash i} m_{k \rightarrow j}\left(\mathbf{x}_{j}\right),
$$

where $N_{v}(j) \backslash i$ means the neighboring valid nodes of the $j$ th node except the $i$ th node. 
The hidden random variable $\mathbf{x}_{i}$ is computed by summation over the possible states as

$$
\mathbf{x}_{i}=\sum_{\mathbf{x}_{i}} \mathbf{x}_{i} b\left(\mathbf{x}_{i}\right),
$$

where $b\left(\mathbf{x}_{i}\right)$ is the marginal posterior probability (belief) for the $i$ th node.

\subsection{State Inference}

We used mean-shift belief propagation (MSBP) [9] [15] to efficiently estimate the state (or belief) of each node. We only evaluate within a discrete local regular grid of samples centered of the previous state because it is hard to evaluate all the possible states of the hidden variable. The message passing is performed to compute a belief for each sample at one iteration. After computing beliefs of all samples, it obtains a new estimated state by doing mean-shift on the samples. It repeats this process until there is no state change. A reader, who want to look for detailed description about the MSBP, refers to the MSBP [9].

\section{The Proposed Object Tracking Algorithm}

A node is valid if the validity compatibility function $\phi_{v}\left(\mathbf{x}_{i}, v_{i}\right)$ is greater than a threshold value. Otherwise, the node is invalid. If the invalid nodes are used for message passing, we may reach the wrong result in the state inference. To reduce the influence of such a wrong propagation, we propose to reconfigure the graphical structure of the DMRF model by pruning invalid nodes, recovering the nodes that are valid frame but were invalid in the previous frame, and reconnecting the neighboring valid nodes of the pruned invalid node. This reconfiguration forces the graphical structure of the DMRF model to be strongly connected.

Fig. 3 shows a typical example of the reconfiguration of the graphical structure of the DMRF model, where (a) represents that two nodes $V_{j}$ and $V_{o}$ are connected to each other due to the invalid node $V_{k}$ and the invalid node $V_{k}$ only receives messages from the two nodes $V_{j}$ and $V_{o}$, and (b) represents three different situations: (1) two nodes $V_{q}$ and $V_{o}$ are connected to each other due to the invalid node $V_{r}$ and the invalid node $V_{r}$ only receives messages from the two nodes $V_{q}$ and $V_{o}$, (2) four pairs of two nodes $V_{q}$ and $V_{m}, V_{m}$ and $V_{j}, V_{j}$ and $V_{o}$ and $V_{o}$ and $V_{q}$ are connected to each other due to the invalid node $V_{n}$ and the invalid node $V_{n}$ only receives the messages from the four nodes $V_{q}, V_{m}, V_{j}$ and $V_{o}$ and (3) the node $V_{k}$ is recovered to the valid node and the recovered node $V_{k}$ can send messages to two neighboring nodes $V_{j}$ and $V_{o}$.

Table 1 summarizes our proposed object tracking algorithm.

\section{Experimental Results and Discussion}

\subsection{Databases and Measures}

The proposed object tracking method using the DMRF model was implemented on a Windows PC platform with a $2.83 \mathrm{GHz}$ Intel Core 2 Quad CPU and 8 GB RAM in 


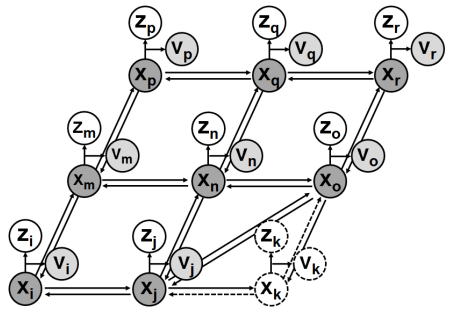

(a) DMRF model at time $\mathrm{t}-1$.

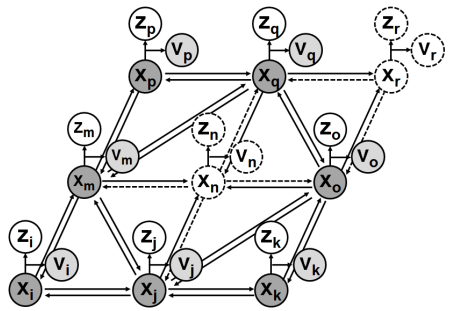

(b) DMRF model at time t.

Fig. 3. A typical example of the graphical structure reconfiguration

Table 1. The proposed object tracking algorithm

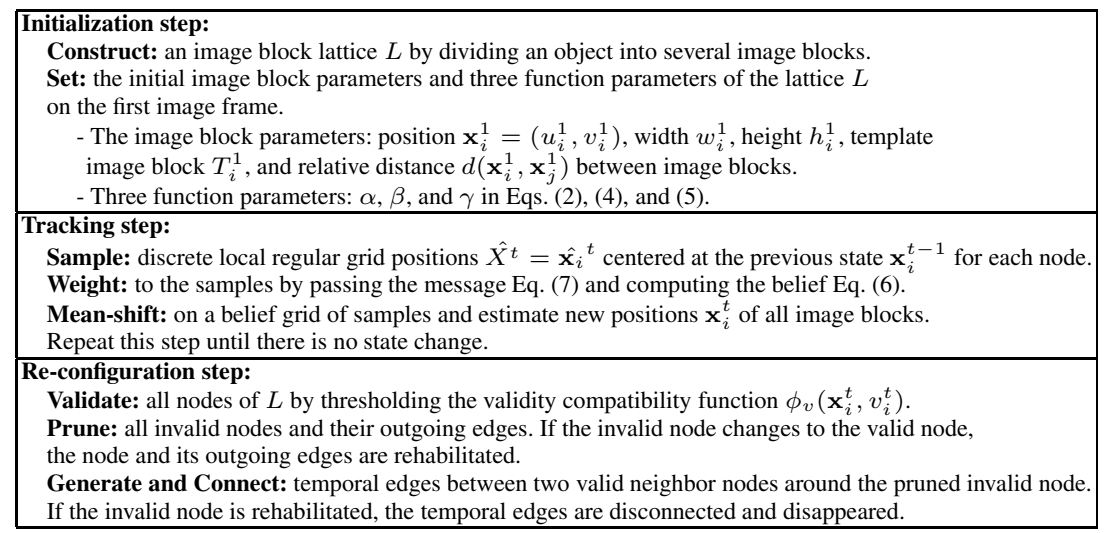

the Matlab 9.0 environment. To evaluate the proposed tracking method, we used 74 different trajectories ( 70 for human and 4 for face) under heavy occlusions. The 70 human trajectories $\left(H_{1}-H_{70}\right)$ were randomly taken from the CAVIAR database 1 , which captured moving people in a a shopping center by a single camera with a resolution of 384 × 288 image and 25 frames per second (fps). Two face trajectories $\left(F_{1}\right.$ and $\left.F_{2}\right)$ were taken from the HONDA database 2 , which captured a moving face by a single camera with a resolution of 640 x 480 image and 15 frames per second (fps). Two face trajectories $\left(F_{3}\right.$ and $\left.F_{4}\right)$ were captured with the Web-camera in our laboratory with a resolution of $320 \times 240$ image and 20 frames per second (fps).

We would like to make a comparison and evaluation on the proposed method (named by 'DMRFT') with the three state-of-the-art tracking methods, which are incremental learning-based tracking method (ILT) [7], semi-supervised learning-based tracking method (SST) [8] and Markov random field-based tracking method (MRFT) [9], through the qualitative and quantitative tracking results. We manually initialized the

\footnotetext{
${ }^{1}$ See http: / / homepages.inf.ed.ac.uk/rbf / CAVIAR

${ }^{2}$ See http://vision.ucsd.edu/ leekc/HondaUCSDVideoDatabase/
} HondaUCSD.html. 
target objects' position and template image block of them at the first frame. We used two block lattice models for MRFT and DMRFT presented in Figure 4 or 5 . We set the parameters $\alpha, \beta$, and $\gamma$ to $0.1,10$, and 1 , respectively.

To evaluate the tracking performance between the tracking results and the ground truth, we consider two measures to evaluate the tracking performance: tracking error and tracking accuracy. Tracking error is the average of the distance between the center point of the tracked object and that of the ground truth during the whole frames. Tracking accuracy is an average of the overlap pixels between the boundary box of the tracked object and that of the ground truth during the whole frames as

$$
\text { Accuracy }=\frac{1}{N} \sum_{t=1}^{N} \frac{R\left(H_{t} \cap G_{t}\right)}{R\left(H_{t} \cup G_{t}\right)},
$$

where $N$ denote the number of frames, $H_{t}$ is the tracked object (bounding box) at the $t$ th frame, $G_{t}$ is the ground truth (bounding box) at the $t$ th frame, and $R(\bullet)$ is a function to compute the area. Generally, we have a good object tracking result if the tracking accuracy is greater than $70 \%$.

\subsection{Quantitative Tracking Results}

Human Tracking. We represented each detected human by the DMRF structure with 20 nodes, where each node corresponded to the center position of template image block. Next, we represented the Euclidean distance between the center positions of two neighboring template image blocks as the edge, which is represented by the white line.

Table 2 (a) summarizes the human tracking results in terms of tracking error among the four different tracking methods. The DMRFT outperformed the three existing methods in terms of average tracking error because it could accurately estimate the center position of the DMRF structure from the successful human tracking of partially visible regions and the successful estimation of occluded regions. Table 2 (b) summarizes the human tracking results in terms of tracking accuracy among the four different tracking methods. The DMRFT outperformed the three existing methods in terms of average tracking accuracy because it could keep the DMRF structures of the occluded humans by the successful tracking of partially visible regions and the successful estimation of occluded regions. The average human tracking accuracy of DMRFT using the 70 human trajectories $H_{1}$ to $H_{70}$ was $72.1 \%$, which is accepted as a good tracking performance.

Face Tracking. We represented each detected face by a DMRF structure with 26 nodes, where each node corresponds to the center position of template image block. Next, we represented the Euclidean distance between the center positions of two neighboring template blocks as the edge, which is represented by the white line.

Table 3 (a) summarizes the face tracking results in terms of tracking error among the four different tracking methods. The DMRFT outperformed the three existing methods in terms of tracking error because it could accurately estimate the center position of the DMRF structure from the successful face tracking of partially visible regions and the successful estimation of occluded regions. The MRFT has moderate face tracking errors except for the face trajectory $F_{4}$ because the tracked faces had not drifted under 
Table 2. Comparison of human tracking errors and accuracies using the human trajectories $H_{1}$ to $H_{70} . E_{H_{1}}$ (or $A_{H_{1}}$ ) and $E_{H_{34}}$ (or $A_{H_{34}}$ ) denote the tracking error (or accuracy) of the human trajectory $H_{1}$ and the tracking error (or accuracy) of the human trajectory $H_{34}$, and $E_{H_{A V G}}$ (or $A_{H_{A V G}}$ ) denotes the average tracking error (or accuracy) of all human trajectories $H_{1}$ to $H_{70}$.

(a) Tracking error

\begin{tabular}{|c|c|c|c|}
\hline \multirow{2}{*}{ Tracking Method } & \multicolumn{3}{|c|}{ Tracking Error } \\
\cline { 2 - 4 } & $E_{H_{1}}$ & $E_{H_{34}}$ & $E_{H_{A V G}}$ \\
\hline ILT [7] & 7.0 & 8.3 & 33.7 \\
SST [8] & 8.9 & 146.1 & 152.2 \\
MRFT [9] & 1.1 & 5.1 & 19.1 \\
DMRFT & 1.0 & 1.3 & 3.4 \\
\hline
\end{tabular}

(b) Tracking accuracy

\begin{tabular}{|c|c|c|c|}
\hline \multirow{2}{*}{ Tracking Method } & \multicolumn{3}{|c|}{ Tracking Accuracy } \\
\cline { 2 - 4 } & $A_{H_{1}}$ & $A_{H_{34}}$ & $A_{H}$ \\
\hline ILT [7] \\
SST [8] & $25.1 \%$ & $34.6 \%$ & $27.5 \%$ \\
MRFT [9] & $35.0 \%$ & $31.5 \%$ & $29.5 \%$ \\
DMRFT & $61.5 \%$ & $57.6 \%$ & $58.4 \%$ \\
& $70.9 \%$ & $73.4 \%$ & $72.1 \%$ \\
\hline
\end{tabular}

heavy occlusion. Table 3 (b) summarizes the face tracking results in terms of tracking accuracy among the four different tracking methods. The DMRFT outperformed the three existing methods in terms of tracking accuracy because it could keep the DMRF structures of the occluded faces by the successful face tracking of partially visible regions and the successful estimation of occluded regions. The face tracking accuracies of DMRFT using the four face trajectories $F_{1}$ to $F_{4}$ were greater than $70 \%$, which are accepted as good tracking performance.

Table 3. Comparison of face tracking errors and accuracies using the human trajectories $F_{1}$ to $F_{4}$. $E_{F_{1}}$ to $E_{F_{4}}$ (or $A_{F_{1}}$ to $A_{F_{4}}$ ) denote the face tracking error (or accuracy) of the face trajectory $F_{1}$ to $F_{4}$, respectively, and $E_{F_{A V G}}$ (or $A_{F_{A V G}}$ ) denotes the average face tracking error (or accuracy) of all the face trajectories $F_{1}$ to $F_{4}$.

(a) Tracking error

\begin{tabular}{|c|c|c|c|c|c|}
\hline \multirow{2}{*}{ Tracking Method } & \multicolumn{5}{|c|}{ Tracking Error } \\
\cline { 2 - 6 } & $E_{F_{1}}$ & $E_{F_{2}}$ & $E_{F_{3}}$ & $E_{F_{4}}$ & $E_{A V G}$ \\
\hline ILT 77] & 22.8 & 1.95 & 17.4 & 31.5 & 23.9 \\
SST [8] & 50.0 & 77.79 & 63.1 & 152.9 & 102.9 \\
MRFT [9] & 6.4 & 1.5 & 1.0 & 33.5 & 15.8 \\
DMRFT & 3.4 & 1.2 & 0.7 & 1.6 & 1.3 \\
\hline
\end{tabular}

(b) Tracking accuracy

\begin{tabular}{|c|c|c|c|c|c|}
\hline \multirow{2}{*}{ Tracking Method } & \multicolumn{5}{|c|}{ Tracking Accuracy } \\
\cline { 2 - 6 } & $A_{F_{1}}$ & $A_{F_{2}}$ & $A_{F_{3}}$ & $A_{F_{4}}$ & $A_{F_{A V G}}$ \\
\hline ILT [7 & $60.6 \%$ & $61.0 \%$ & $19.8 \%$ & $14.2 \%$ & $20.0 \%$ \\
SST [8 & $35.0 \%$ & $41.7 \%$ & $51.1 \%$ & $17.2 \%$ & $34.9 \%$ \\
MRFT [9] & $70.8 \%$ & $66.9 \%$ & $68.8 \%$ & $25.4 \%$ & $49.5 \%$ \\
DMRFT & $74.9 \%$ & $72.6 \%$ & $71.6 \%$ & $72.7 \%$ & $72.3 \%$ \\
\hline
\end{tabular}

\subsection{Qualitative Tracking Results}

Human Tracking Examples. Fig. 4 shows the human tracking results of the human trajectory $H_{1}$ and $H_{34}$ over three different frames, respectively, where the black and white block in the case of (d)(i) and (e)(j) of two figures denote valid and invalid blocks, respectively. The tracking results using ILT and SST failed to track the occluded human because the bounding box had shrunk, drifted or disappeared. The tracking results using MRFT missed the occluded human because the human structure had drifted onto the occluding human due to the occlusion. On the other hand, the tracking results using DMRFT succeeded in tracking the occluded human because it tracked the partially visible regions well and was almost unaffected by the occluded regions.

Face Tracking Examples. Fig. 5 shows the face tracking results of the face trajectory $F_{2}$ and $F_{4}$ over three different frames, respectively, where the black and white block in 
the case of (d)(i) and (e)(j) of two figures denote valid and invalid blocks, respectively. The tracking results using ILT and SST failed to track the occluded face because the bounding box had drifted, shrunk or disappeared. The tracking results using MRFT in the Fig. 5 (d) was good, but those in the Fig. 5(i) missed the occluded face because the MRF structure of the occluded face had drifted. On the other hand, the tracking results using DMRFT succeeded in tracking the occluded face although the occluding hand passed over the face.

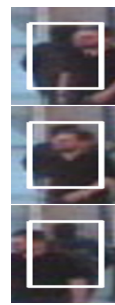

(a)

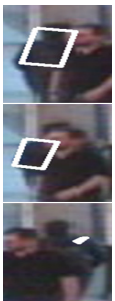

(b)

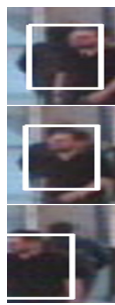

(c)

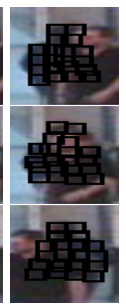

(d)

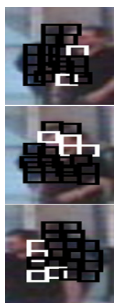

(e)

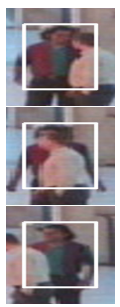

(f)

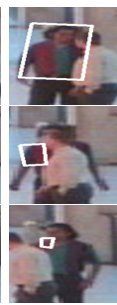

(g)

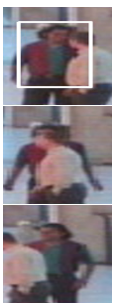

(h)

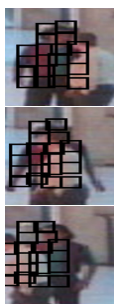

(i)

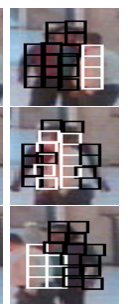

(j)

Fig. 4. Human tracking results of the human trajectory $H_{1}(\mathrm{a} \sim \mathrm{e})$ and $H_{34}(\mathrm{f} \sim \mathrm{j})$. (a)(f) GT, (b)(g) ILT, (c)(h) SST, (d)(i) MRFT, and (e)(j) DMRFT.

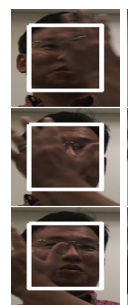

(a)

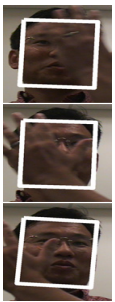

(b)

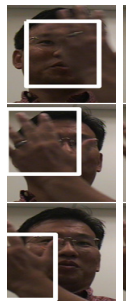

(c)

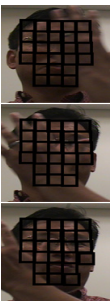

(d)

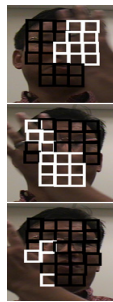

(e)

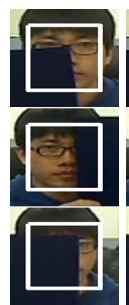

(f)

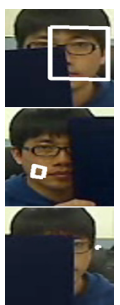

(g)

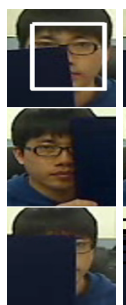

(h)

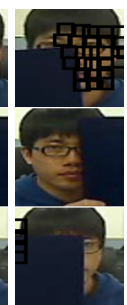

(i)

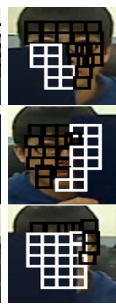

(j)

Fig. 5. Face tracking results of the face trajectory $F_{2}(\mathrm{a} \sim \mathrm{e})$ and $F_{4}(\mathrm{f} \sim \mathrm{j}) .(\mathrm{a})(\mathrm{f}) \mathrm{GT}$, (b)(g) ILT, (c)(h) SST, (d)(i) MRFT, and (e)(j) DMRFT.

\section{Conclusion}

We proposed a new dynamic Markov random field (DMRF) model for tracking an object under severe occlusion. In the visual tracking problem, the conventional Markov random field (MRF) and belief propagation (BP) tackle a multi-modal posterior distribution problem, but it is vulnerable for tracking an heavily occluded object due to wrong propagations from invalid nodes. Especially, their use produces a global drift and distortion of the whole layout with an image block lattice model. However, DMRF, which is a bidirectional graph which consists of three random variables:hidden, observation, and validity, reduce the effect of such wrong propagation by temporally pruning invalid nodes and linking edges among valid nodes. The proposed object tracking method using the DMRF surprisingly tracks a heavily occluded object even though experimenting on various object sequences with severe occlusion. 
Acknowledgments. This work was supported by the R\&D program of Ministry of Culture, Sports and Tourism (MCST) and Korea Evaluation Institute of Industrial Technology (KEIT) (10039923, Development of Live4D contents platform technology based on expansion of realistic experiential space).

\section{References}

1. Wu, B., Nevatia, R.: Detection and segmentation of multiple, partially occluded objects by grouping, merging, assigning part detection responses. International Journal of Computer Vision 82, 185-204 (2009)

2. Senior, A., Hampapur, A., Tian, Y., Brown, L.: Appearance models for occlusion handling. Image and Vision Computing 24, 1233-1243 (2006)

3. Hu, W., Zhou, X., Hu, M., Maybank, S.: Occlusion reasoning for tracking multiple people. IEEE Transactions on Circuits and Systems for Video Technology 19, 114-121 (2009)

4. Zhao, T., Nevatia, R.: Tracking multiple humans in complex situations. IEEE Transactions on Pattern Analysis and Machine Intelligence 26, 1208-1221 (2004)

5. Mittal, A., Davis, L.S.: M2tracker: A multi-view approach to segmenting and tracking people in a cluttered scene using region-based stereo. International Journal of Computer Vision 51, 189-203 (2003)

6. Lin, Z., Davis, L., Doermann, D., Dementhon, D.: An interactive approach to pose-assisted and appearance-based segmentation of humans. In: Proc. of IEEE International Conferenece on Computer Vision, pp. 1-8 (2007)

7. Ross, D., Lim, J., Lin, R.: Incremental learning for robust visual tracking. International Journal of Computer Vision 77, 125-141 (2008)

8. Mei, X., Ling, H.: Beyond semi-supervised tracking: Tracking should be as simple as detection, but not simpler than recognition. In: Proc. of IEEE International Conferenece on Computer Vision Workshop, pp. 1409-1416 (2009)

9. Park, M., Liu, Y., Collins, R.: Efficient mean shift belief propagation for vision tracking. In: Proc. of IEEE Computer Vision and Pattern Recognition, pp. 1-8 (2008)

10. Lin, W., Liu, Y.: A lattice-based mrf model for dynamic near-regular texture tracking. IEEE Transactions on Pattern Analysis and Machine Intelligence 29, 777-792 (2007)

11. Liu, J., Liu, Y.: Multi-target tracking of time-varying spatial patterns. In: Proc. of IEEE Computer Vision and Pattern Recognition, pp. 1839-1846 (2010)

12. Coughlan, J., Huiying, S.: Shape matching with belief propagation: Using dynamic quantization to accomodate occlusion and clutter. In: Proc. of IEEE Computer Vision and Pattern Recognition Workshop (2004)

13. Felzenszwalb, P., Huttenlocher, D.: Efficient belief propagation for early vision. International Journal of Computer Vision 70, 41-54 (2006)

14. Yedidia, J., Freeman, W., Weiss, Y.: Understanding belief propagation and its generalizations. In: International Joint Conference on Artificial Intelligence (2001)

15. Park, M., Brocklehurst, K., Collins, R., Liu, Y.: Deformed lattice detection in real-world images using mean-shift belief propagation. IEEE Transactions on Pattern Analysis and Machine Intelligence 31, 1804-1816 (2009) 